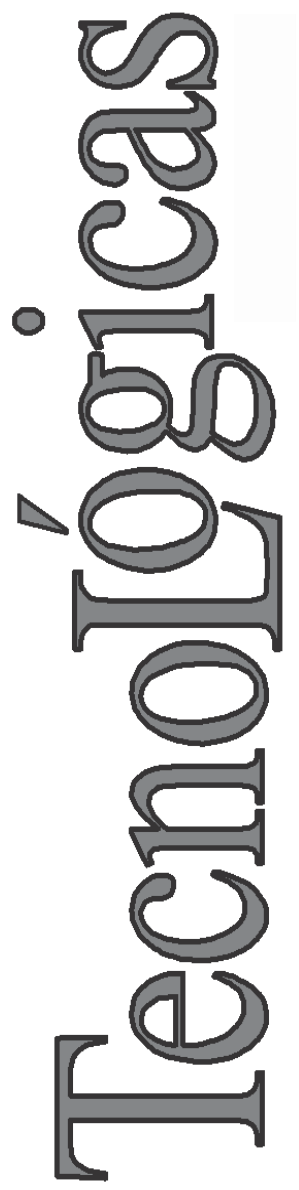

\title{
Clasificación de Mariposas por Modelos de Color HSI y RGB Usando Redes Neuronales
}

\section{Butterfly Classification by HSI and RGB Color Models Using Neural Networks}

Jorge E. Grajales-Múnera ${ }^{1}$ Alejandro Restrepo-Martínez ${ }^{2}$

1 Estudiante del Programa de Ingeniería de Sistemas, Facultad de Ingeniería, Instituto Tecnológico Metropolitano, Medellín-Colombia jorgegrajales17501@correo.itm.edu.co 2 Docente-Investigador del Programa de Ingeniería de Sistemas, Facultad de Ingeniería, Instituto Tecnológico Metropolitano, Medellín-Colombia alejandromartinez@itm.edu.co 


\title{
Resumen
}

Redes Neuronales Artificiales y el procesamiento de imágenes. Se procesan imágenes de un total de 9 especies de mariposas del género Morpho el cual tiene el azul como color característico. Para realizar la segmentación de la mariposa se usan herramientas de procesamiento de imágenes tales como: binarización, filtrado de bordes y morfología matemática. Para el procesamiento de los datos se obtienen los datos RGB de cada imagen que se llevan al modelo de color HSI con el objetivo de identificar los pixeles que son de color azul y de obtener los datos para realizar el procesamiento en las Redes Neuronales propuestas: Back-propagation y perceptrón. Para la verificación y análisis de resultados se construyen matrices de confusión, se analizan y se toman los resultados de las redes neuronales que muestren los niveles de error más bajos. Se logra llegar a un nivel de error cercano al 1\% en la clasificación en algunas especies de mariposas.

\section{Palabras clave} lor.

Perceptrón; back-propagation; mariposa; redes neuronales; análisis del co-

\begin{abstract}
This study aims the classification of Butterfly species through the implementation of Neural Networks and Image Processing. A total of 9 species of Morpho genre which has blue as a characteristic color are processed. For Butterfly segmentation we used image processing tools such as: Binarization, edge processing and mathematical morphology. For data processing RGB values are obtained for every image which are converted to HSI color model to identify blue pixels and obtain the data to the proposed Neural Networks: Back-Propagation and Perceptron. For analysis and verification of results confusion matrix are built and analyzed with the results of neural networks with the lowest error levels. We obtain error levels close to $1 \%$ in classification of some Butterfly species.
\end{abstract}

\section{Keywords}

Perceptron; back-propagation; neural networks; butterfly; color analysis. 


\section{INTRODUCCIÓN}

En general las metodologías de clasificación de mariposas se abordan con uno o con la combinación de varios de los siguientes métodos: 1. El análisis de la forma de las alas. 2. El análisis de la textura de las alas. 3. El análisis del color de las alas. Kang, Song y Lee (2012) abordan el proceso de identificación mediante el reconocimiento de las formas de las alas de las mariposas a partir de un método llamado entropía BLS (Branch Length Similarity). Los datos generados por este método son los datos de entrada para una red Back-Propagation de tres capas para procesar imágenes de 7 especies de mariposas. En Kaya et al. (2013) y Kaya 2013-2 abordan la identificación de especies en base a las características de la textura de las alas de las mariposas. La extracción de la información relevante de las mariposas se realiza aplicando los filtros de Gabor para posteriormente procesar los datos con un tipo de red llamada ELM (Extreme Learning Machine) ó Maquinas de Aprendizaje Extremo.

En Kaya y Kayci (2013) se propone un método basado en redes neuronales artificiales y el procesamiento de imágenes en base a la textura y el color de las alas de las mariposas logrando llegar un nivel del $92.85 \%$ de exactitud en la clasificación. Otra aproximación como la de Piszter, Kertész, Vértesy, Bálin y Biró (2011) realiza la clasificación mediante una Red Neuronal de un total de 9 especies de mariposas azules Lycaenid utilizando el espacio de color CIE 1931. Con la mitad de los especímenes se realiza el entrenamiento mientras que con la otra mitad de los datos se realiza la validación de la red neuronal. Con esta aproximación se logró un $96 \%$ de precisión en la clasificación. También existen aproximaciones sin utilizar Redes Neuronales como la de Bhanu, B., Li, R., Heraty, J., Murray, E., 2008, con un algoritmo basado en un modelo probabilístico llamado Gaussian Mixture Model. En este método las imágenes de las mariposas se dividen en partes y estas son clasificadas con el algoritmo en base a los patrones de textura encontrados.

Como las estructuras y los colores de las alas de las mariposas son complejas de analizar por su amplia variabilidad y patrones irregulares, incluso entre las especies de un mismo género, este 
estudio presenta un procedimiento que implementa y permite hacer una comparativa del desempeño de diferentes redes neuronales con diferentes tipos de aprendizaje y diferentes combinaciones de los datos de color de las imágenes de las mariposas para realizar la clasificación de 9 especies diferentes del género Morpho, con el objetivo de verificar cuál combinación de red neuronal y con qué tipo de dato (RGB ó HSI) existe un mejor desempeño. Se realiza un análisis de los porcentajes de error arrojados por cada red neuronal, además de verificar la información arrojada por las matrices de confusión para verificar el desempeño.

\section{METODOLOGíA}

\subsection{Imágenes de las Mariposas}

Las imágenes de las 9 especies analizadas fueron tomadas de la Fundación Butterflies Of America (http://www.butterfliesofamerica.com) una iniciativa que contiene un catálogo ilustrado "online" de las fotografías de más de 8300 especies de América (ver Fig. 1). Cada imagen tiene un tamaño original de 1120 pixeles de ancho x 800 píxeles de alto. Para realizar el análisis de las imágenes en el algoritmo, cada imagen se reescala con el método de interpolación bilineal a un tamaño de 500 pixeles de ancho x 352 pixeles de alto lo que da un total de 176.000 pixeles procesados por imagen.

\subsection{Herramientas de Desarrollo}

La herramienta de procesamiento de las imágenes se basa en la implementación de los algoritmos en Visual Studio 2012 C\# y el "framework" de código abierto para la implementación de Redes Neuronales y Visión Artificial AForge (http://www.aforgenet.com/) 


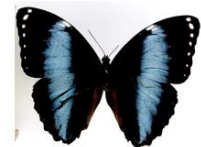

Morpho Achilles
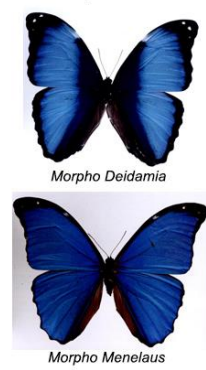

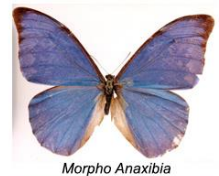

Morpho Anaxibia
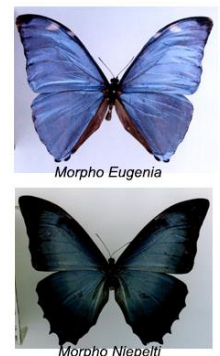
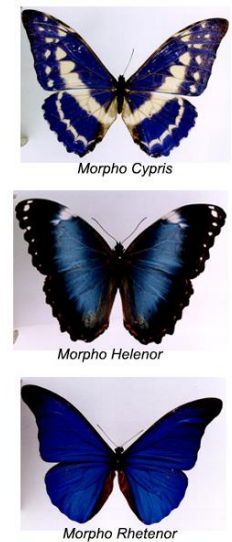

Fig. 1. Imágenes de las 9 especies de mariposas analizadas en el estudio. Tomadas de la fundación Butterflies Of America (http://www.butterfliesofamerica.com)

\subsection{Método Propuesto}

A continuación se describen las fases del algoritmo empleado para realizar el procesamiento de las imágenes y su posterior entrenamiento en la Red Neuronal (ver Fig. 2).

Fase 1: Leer y reescalar la imagen a un tamaño 500 x 352 pixeles.

Fase 2: Se extrae la región de interés mediante un filtro de bordes, luego se hace una operación morfológica de dilatación con elemento estructurante en forma de diamante de tamaño $5 \mathrm{x} 5$ para rellenar el interior de los bordes y finalmente se aplica una operación lógica AND.

Fase 3: Extraer los datos RGB de la imagen que luego se llevan al espacio de color HSI.

Fase 4: Se llenan las matrices de entrenamiento con los datos RGB y HSI. También se llena la matriz de los datos esperados por cada pixel, los cuales son calculados en base al tono y la saturación.

Fase 5: Entrenamiento de las redes neuronales de perceptrón y Back-Propagation con los datos RGB y HSI.

Fase 6: Análisis de los resultados de la clasificación. (Matrices de Confusión). 


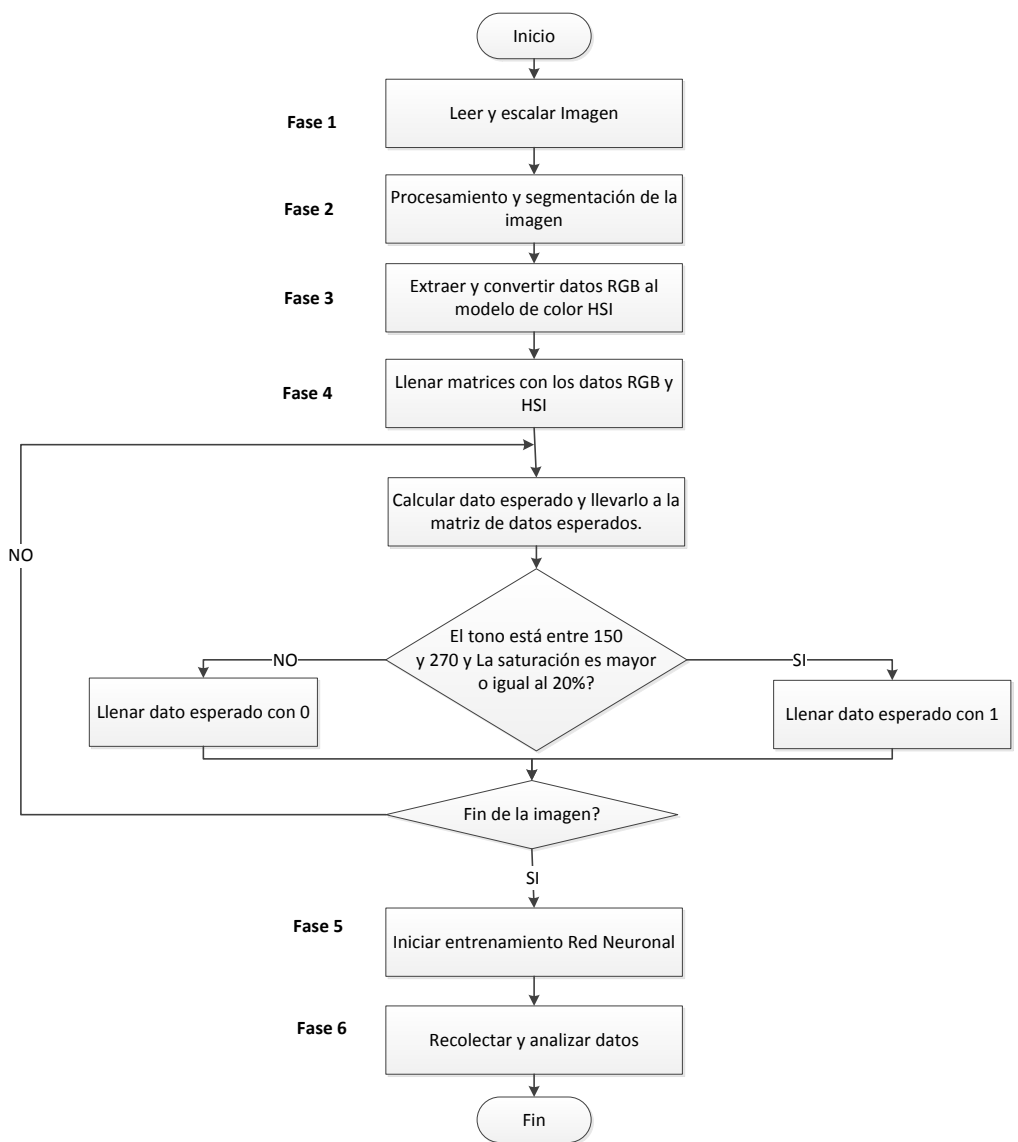

Fig. 2. Diagrama de flujo del algoritmo que procesa los datos de las imágenes de las mariposas

\subsubsection{Segimentación y extracción de los datos}

El primer paso consiste en extraer los bordes de la Mariposa, para ello se aplica el filtro basado en gradiente de Sobel. El segundo paso consiste en aplicar la operación morfológica de dilatación con elemento estructurante en forma de diamante para rellenar y juntar los espacios entre bordes los cuales contienen los colores de las alas de la Mariposa. Finalmente se aplica operador AND de la primera imagen con el resultado de la dilatación para realizar la separación final del fondo y de la Mariposa. 
La red neuronal sólo procesa los píxeles que pertenecen a la mariposa con el objetivo de buscar los pixeles de color azul. El cálculo de la matriz de los datos esperados de la matriz se hace en base al tono y a la saturación de cada pixel. Si el tono está en un rango de azules (entre 150 y 270) y la saturación está en el rango mayor del $20 \%$ (con el objetivo de excluir los blancos) se clasifica el valor esperado en 1 , si no se clasifica en 0 .

\subsubsection{Implementación de las redes neuronales}

Inicialmente se ha comenzado con el entrenamiento de la red neuronal de tipo perceptrón simple y luego con la red neuronal de tipo Back-Propagation. Los tres datos de entrada son el valor del pixel en R, en G y en B. En el caso del modelo de color HSI los datos de entrada son el valor del pixel en $\mathrm{H}$, en S, y en I.

Perceptrón simple: Para la red neuronal perceptrón se tiene la siguiente estructura: Tres datos de entrada y uno de salida. Los tres pesos (w1, w2, w3) se inicializan con valores aleatorios. La red tiene una sola capa y una sola neurona. La función de activación es: $f(x)=\left\{\begin{array}{l}1 \text { si } \boldsymbol{w} \cdot \boldsymbol{x}-\boldsymbol{u}>0 \\ 0 \text { en otro caso }\end{array}\right.$ El índice de aprendizaje es de 0.1. Se realizan 500 épocas para el aprendizaje. Para el entrenamiento se usa el 100\% de los datos y para la validación se usan el 100\% de los datos aplicando los pesos finales del entrenamiento.

Back-Propagation: Para la red neuronal Back-Propagation se tiene la siguiente estructura: Tres datos de entrada y uno de salida. Los pesos se inicializan con valores aleatorios. La red neuronal tiene tres capas con una neurona cada una. Se usa la función de activación sigmoide. El índice de aprendizaje es de 0.1. Se realizan 500 épocas para el aprendizaje Para el entrenamiento se usa el $100 \%$ de los datos y para la validación se usan el 100\% de los datos aplicando los pesos finales del entrenamiento.

\section{RESULTADOS Y DISCUSIÓN}

Con el objetivo de conocer la red que presenta un mayor de nivel de confiabilidad con respecto a los valores de error, se muestran un conjunto de tablas comparativas: En la Tabla 1 se muestran los errores de entrenamiento de ambas redes: perceptrón y 
Back-Propagation por cada una de las 9 especies con los datos RGB de las imágenes con el $100 \%$ de los datos. En la Tabla 2 se muestran los errores de entrenamiento con el $100 \%$ de los datos con los datos HSI de las imágenes.

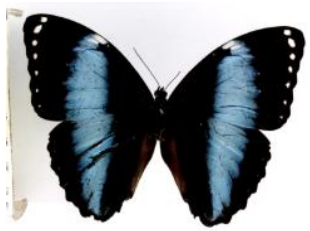

4

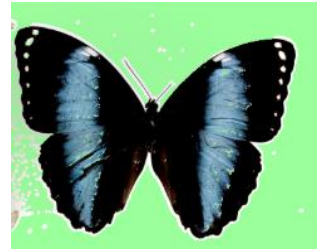

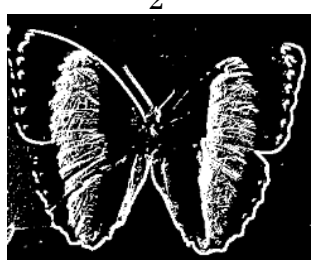

5

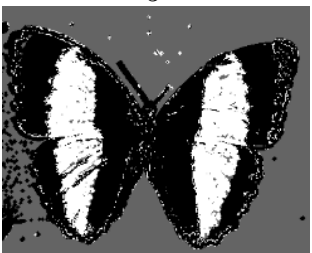

3

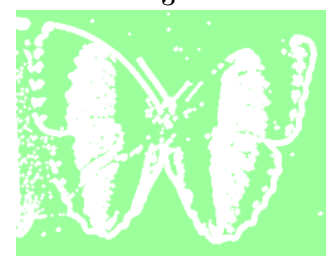

Fig. 3. Imagen original de la especie "Morpho Achilles" (1). El resultado de aplicar el Filtro de Sobel (2). Imagen resultado de la Operación de dilatación (3). Imagen resultado de aplicar el operador AND entre 1 y 3 (4) Imagen con los datos de entrenamiento (5)

Tabla 1. Error total y porcentaje de error del entrenamiento de cada una de las especies de mariposas con los datos RGB

\begin{tabular}{lcccc}
\hline & \multicolumn{2}{c}{ Perceptrón } & \multicolumn{2}{c}{ Back-Propagation } \\
\hline & $\begin{array}{c}\text { Pixeles mal } \\
\text { clasificados }\end{array}$ & Error (\%) & $\begin{array}{c}\text { Pixeles mal } \\
\text { clasificados }\end{array}$ & Error (\%) \\
\hline Morpho achilles & 620 & 1,51 & 701,223 & 1,71 \\
Morpho anaxibia & 2439 & 4,68 & 2109 & 4,04 \\
Morpho cypris & 2225 & 3,47 & 1285,29 & 2,01 \\
Morpho deidamia & 5453 & 6,42 & 3302,39 & 3,89 \\
Morpho eugenia & 6625 & 5,51 & 3504,31 & 2,91 \\
Morpho helenor & 992 & 2,27 & 445,235 & 1,02 \\
Morpho menelaus & 1993 & 2,44 & 3988,37 & 4,89 \\
Morpho niepelti & 621 & 1,14 & 600,7395 & 1,10 \\
Morpho rhetenor & 1571 & 2,61 & 2522,226 & 4,19 \\
\hline \multicolumn{1}{c}{ Total } & 22539,00 & 30,05 & 18458,78 & 25,76 \\
\hline \multicolumn{1}{c}{ Promedio } & 2504,33 & 3,34 & 2050,98 & 2,86 \\
\hline
\end{tabular}


Tabla 2. Error total y porcentaje de error del entrenamiento de cada una de las especies de mariposas con los datos HSI

\begin{tabular}{|c|c|c|c|c|}
\hline & \multicolumn{2}{|c|}{ Perceptrón } & \multicolumn{2}{|c|}{ Back-Propagation } \\
\hline & $\begin{array}{l}\text { Pixeles mal } \\
\text { clasificados }\end{array}$ & Error (\%) & $\begin{array}{l}\text { Pixeles mal } \\
\text { clasificados }\end{array}$ & $\begin{array}{c}\text { Error } \\
(\%)\end{array}$ \\
\hline Morpho achilles & 1288 & 3,14 & 1381,115 & 3,36 \\
\hline Morpho anaxibia & 2961 & 5,68 & 2102,7923 & 4,03 \\
\hline Morpho cypris & 4250 & 6,63 & 4756 & 7,42 \\
\hline Morpho deidamia & 4922 & 5,79 & 3453,34 & 4,07 \\
\hline Morpho eugenia & 5867 & 4,88 & 4733,59 & 3,94 \\
\hline Morpho helenor & 1538 & 3,52 & 910,026 & 2,08 \\
\hline Morpho menelaus & 3526 & 4,32 & 5349,367 & 6,55 \\
\hline Morpho niepelti & 2378 & 4,36 & 1337 & 2,45 \\
\hline Morpho rhetenor & 2062 & 3,43 & 1685,54 & 2,80 \\
\hline Total & 28792 & 41,74 & 25708,77 & 36,70 \\
\hline Promedio & 3199,11 & 4,64 & 2856,53 & 4,08 \\
\hline
\end{tabular}

Con una media de $2,86 \%$ en el porcentaje de error, la red neuronal con Back-Propagation de los datos RGB muestra el mejor desempeño en la clasificación. Mientras que el perceptrón con el $100 \%$ de los datos con los datos HSI muestra el peor desempeño con una media de 4,64\% entonces el modelo RGB funcionó mejor en la clasificación.

Los mejores desempeños de redes individuales se encuentran en la Red Neuronal con los datos de la la Morpho helenor con el $1.02 \%$ y la Morpho niepelti con el $1.10 \%$. Para estas dos redes se construyen dos Matrices de Confusión, se pueden ver en las Tablas 3 y 4 para el Perceptrón y en las Tablas 5 y 6 con los datos de Back-Propagation.

Tabla 3. Matriz de confusión de la especie "Morpho helenor" y "Morpho niepelti" con el Perceptrón y los datos RGB

\begin{tabular}{ccc|ccc}
\hline \multicolumn{2}{c}{ Matriz de Confusión } & Morpho helenor & \multicolumn{3}{|c}{ Matriz de Confusión } \\
\hline & 0 & 1 & & 0 & 1 \\
0 & 21362 & 487 & 0 & 29988 & 313 \\
1 & 496 & 21353 & 1 & 311 & 26990 \\
\hline
\end{tabular}

Las matrices de confusión muestran un buen equilibrio de datos en la clasificación salvo la especie "Morpho Niepelti" con la Red Neuronal Back-Propagation. Se presenta una confusión de la red 
con respecto a los pixeles que deberían ser diferentes del azul y que los está clasificando como si fueran azules.

Tabla 4. Matriz de confusión de la especie "Morpho helenor" y "Morpho niepelti" con Back-Propagation y los datos RGB

\begin{tabular}{ccc|ccc}
\hline \multicolumn{2}{c}{ Matriz de Confusión } & Morpho helenor & \multicolumn{3}{c}{ Matriz de Confusión Morpho niepelti } \\
\hline & 0 & 1 & & 0 & 1 \\
\hline 0 & 21298 & 249 & 0 & 23951 & 153 \\
\hline 1 & 551 & 21600 & 1 & 3350 & 27148 \\
\hline
\end{tabular}

Con respecto al modelo de color HSI, analizando los pesos finales de las Redes Neuronales se evidencia que la $\mathrm{H}$ (Tono) no tiene mucha relevancia comparado con la saturación (299.5 del Tono y 36125.53 de la Saturación) Se puede decir que la red neuronal no está siendo capaz de clasificar el tono apropiadamente. En la Tabla 5 se pueden ver los datos de los pesos finales H, S e I.

Tabla 5. Pesos finales de la red neuronal del perceptrón con los datos HSI

\begin{tabular}{lccc}
\hline & \multicolumn{3}{c}{ Perceptrón } \\
\hline & Peso Final H & Peso Final S & Peso Final I \\
\hline Morpho achilles & 17.56497634 & 645.2979269 & 17.16581746 \\
Morpho anaxibia & 30.92650008 & 1389.754713 & -13.43787574 \\
Morpho cypris & 66.29504519 & 7947.919711 & 2.710030684 \\
Morpho deidamia & 71.29896993 & 6896.501208 & -27.27387531 \\
Morpho eugenia & -19.33829792 & 12710.08751 & -41.84824988 \\
Morpho helenor & 24.48488701 & 1126.408171 & 27.6737065 \\
Morpho menelaus & 29.14433489 & 1014.813303 & 90.10111919 \\
Morpho niepelti & 54.26802862 & 491.1945732 & -0.630845755 \\
Morpho rhetenor & 24.88011256 & 3903.559714 & -10.53900637 \\
\hline \multicolumn{1}{c}{ Total } & 299.5245567 & 36125.53683 & 43.92082078 \\
\hline
\end{tabular}

\section{CONCLUSIONES}

En el análisis de los resultados se logra detectar un mejor desempeño de las redes neuronales basadas en los datos RGB de las imágenes de las especies de mariposas ya que en las redes neuronales con los datos HSI se ven porcentajes de error mayores al 5\% en 3 especies en el caso del perceptrón y 2 en el caso de Back-Propagation (Tabla 2) mientras que en las redes neuronales 
con los datos en RGB sólo 2 especies presentan un porcentaje de error mayor al 5\% (Tabla 1). Se puede decir que Las Redes Neuronales con Back-Propagation son las que muestran un mejor desempeño basados en el porcentaje de error.

Se logran obtener unos porcentajes de error bajos en dos de las especies aunque las matrices de confusión dejan una duda de la confiabilidad con respecto a una de ellas. Para confirmar o descartar este resultado se hace necesario implementar otros tipos de entrenamiento y validación además de hacer varias modificaciones en la estructura de las redes de tipo Back-Propagation con respecto a las topologías de red y verificar si se pueden obtener mejores resultados.

\section{REFERENCIAS}

Bhanu, B., Li, R., Heraty, J., Murray, E., 2008. Automated classification of skippers based on parts representation. Am. Entomol. 54, 228-231.

Kang, S.H., Song, S.H., Lee, S.H., 2012, Identification of butterfly species with a single neural network system, Journal of Asia-Pacific Entomology, p. 431.

Kang, S.H., Wonju, J., Lee, S.H., 2012, Butterfly species identification by branch length similarity entropy, Journal of Asia-Pacific Entomology, p. 437.

Kaya, Y., Kayci, L., Tekin, R., 2013, A Computer Vision System for the Automatic Identification of Butterfly Species via Gabor-Filter-Based Texture Features and Extreme Learning Machine: GF+ELM, TEM Jour-nal - Volume 2 / Number 1.

Kaya, Y., Kayci L., 2013, Application of artificial neural network for automatic detection of butterfly species using color and texture features, Springer-Verlag Berlin Heidelberg 2013.

Piszter, G., Kertész K.., Vértesy Z., Bálint, Z., Biró, L.P., 2011 Photonic Nanoarchitectures in Butterfly Scales Allowing Species Identification, Procedia Computer Science 7 (2011) p. 200-201.

González R.C., Woods R.E., 1996, Tratamiento Digital de Imágenes, Adison-Wesley Publishing Co. 1996. 